BBA 95012

\title{
STUDIES ON THE INHIBITION OF BACILLUS SUBTILIS GROWTH BY CORDYCEPIN
}

\author{
FRITZ ROTTMAN* AND ARMAND J. GUARINO** \\ Department of Biological Chemistry, \\ University of Michigan, Ann Avbor, Mich. (U.S.A.)
}

(Received October 28th, 1963)

\begin{abstract}
SUMMARY
I. This work was undertaken to determine the specific enzymatic site at which cordycepin inhibits the growth of Bacillus subtilis. Of a number of compounds tested, the nucleosides of adenine and guanine were most effective in reversing this inhibition. This fact, coupled with the failure to demonstrate the accumulation of ultravioletabsorbing intermediates in the acid-soluble fraction of these cells, as well as the ability of 5-amino-4-imidazolecarboxamide to partially reverse the inhibition of growth, suggests that cordycepin exerts its growth inhibitory effect in this organism on reactions in the synthesis of purines de novo.

2. Bacillus subtilis is capable of cleaving cordycepin to adenine and cordycepose. The fact that adenine can reverse cordycepin inhibition probably best explains the ability of this organism to overcome the inhibition at low concentrations.

3. Replacement of the 6-amino group of cordycepin by a hydroxyl group produced the hypoxanthine analog of cordycepin which was much less effective as an inhibitor.
\end{abstract}

\section{INTRODUCTION}

In I95I, cordycepin, a nucleoside isolated from culture filtrates of the mold Cordyceps militaris was found to inhibit the growth of $B$. subtilis N.C.T.C. strain 6752 (ref. I). More recently this compound was shown to antagonize the growth of the Ehrlich mouse ascites tumor cell in vivo ${ }^{2}$ and when incubated with the same cells in vitro was shown to suppress the uptake of labeled phosphate into nucleic acids ${ }^{3}$. We have been interested in establishing the enzymatic site or sites at which this compound exerts its growth inhibitory effect.

As a first approach to this problem, we chose a strain of $B$. subtilis (ATCC 10783) whose growth is inhibited at relatively low concentrations of cordycepin. Various metabolites were added to growth-inhibited cultures to determine which compounds had the ability to reverse this effect. This report presents the results of such a study, and in a preliminary way implicates pathways of purine biosynthesis as a site of inhibition.

* Present Address: National Institutes of Health, Bethesda, Md. (U.S.A.).

** Present Address: Department of Biochemistry, Woman's Medical College of Pennsylvania, Philadelphia 29, Pa. (U.S.A.). 


\section{METHODS}

Cordycepin was obtained from 30 -day old cultures of $C$. militaris by a published procedure ${ }^{4}$. The final product was recrystallized twice before use. Deoxyadenosine, adenosine, adenine, guanine, deoxyguanosine, inosine, deoxyinosine, uridine, and 5-amino-4-imidazolecarboxamide were purchased from Calbiochem. Guanosine, hypoxanthine, and xanthine were obtained from Schwarz Laboratories. Cytidine was a product of Pabst Laboratories.

Cordycepose, the sugar moiety of cordycepin, was prepared by acid-hydrolysis of cordycepin on Dowex-50 $\left(\mathrm{H}^{+}\right) .25 \mathrm{Img}$ of cordycepin were suspended in $20 \mathrm{ml}$ of water. Approx. $7 \mathrm{ml}$ (packed, wet volume) of Dowex-50 X 8, $\mathrm{H}^{+}$phase, $200-400$ mesh were added to the solution, and the suspension was heated for $\mathrm{I} h$ on a steam bath.

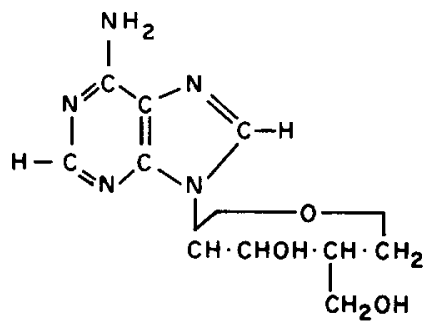

COROYCEPIN

Fig. I.

Following this treatment and removing the resin by centrifugation, less than $0.2 \%$ of the initial ultraviolet-absorbing material was found in the supernatant fraction. On the other hand, roo \% of the cordycepose, as determined by a modified anthrone procedure ${ }^{4}$, still remained in the supernatant fluid. The cordycepose so obtained was used without further purification.

The deaminated product of cordycepin was prepared by a modification of the procedure of LEvene AND Tipson ${ }^{5}$. The process of deamination was allowed to proceed for 3 days at $25^{\circ}$. The final reaction mixture was adjusted to $\mathrm{pH}$ Ir.4 and the product absorbed on a Dowex-I X 8, $\mathrm{Cl}^{-}$resin column, 200-400 mesh. Unreacted cordycepin was eluted from the resin with water adjusted to $\mathrm{pH}$ ro with aqueous ammonia. The resin column was then washed with water ( $\mathrm{pH} \mathrm{6)}$ and finally with o.I $\mathrm{N}$ acetate buffer ( $\mathrm{pH} 4.5$ ), which eluted the deaminated product. The pooled fractions were concentrated on a rotary evaporator at $40^{\circ}$ under vacuum. The absorption spectra of the deaminated product agreed with that of $\mathrm{N}-9$ substituted hypoxanthine in both acid and alkali.

The chemically defined media of HERRIOTT AND BARLOW ${ }^{6}$, containing inorganic salts and glucose, was used in these experiments. Inhibition studies were performed in culture tubes in a final volume of Io $\mathrm{ml}$ to which $0.2 \mathrm{ml}$ of an II-hour culture of $B$. subtilis was added.Growth of the organisms was followed turbidimetrically at $65^{\circ}$ $\mathrm{m} \mu$ in a Bausch and Lomb Spectronic 20 photometer. An initial study was carried out under identical conditions to establish that the observed increase in absorbancy corresponded to an actual increase in the number of viable cells.

Two sources of $B$. subtilis ATCC I0783 (originally classified NRRL strain B543) were used in these studies and both yielded identical results. One of these, classified 
as NRRL strain B543 was kindly supplied by Dr. D. Merchant, Department of Microbiology, The University of Michigan, Ann Arbor, Mich. The other was purchased from the American Type Culture Collection.

\section{RESULTS AND DISCUSSION}

The ability of cordycepin to inhibit growth was tested initially in cultures of Escherichia coli; B. subtilis, Strain So. Michigan; B. subtilis, Strain Marburg; and $B$. subtilis, ATCC 10783. Only the growth of the latter organism was found to be significantly inhibited by cordycepin at concentrations of $\mathrm{I} 0 \mu \mathrm{g} / \mathrm{ml}$. The results of a typical growth inhibitory experiment are shown in Fig. 2. Growth inhibition in the

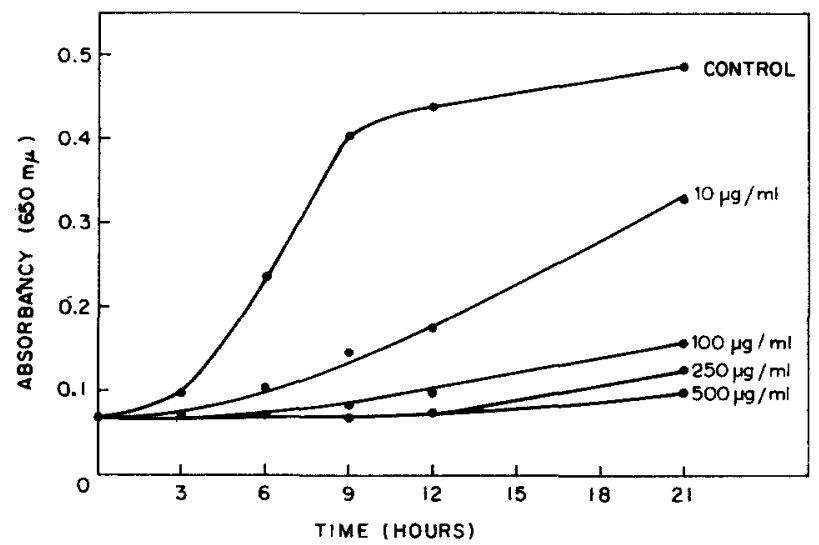

Fig. 2. Inhibition of $B$. subtilis growth by cordycepin. Cordycepin was added before sterilization at the concentrations indicated in the figure. Growth was followed turbidimetrically as described in the text.

presence of low concentrations of cordycepin was partially overcome. In fact, in this figure which follows the growth over $2 \mathrm{I}$ h, the growth of the culture containing ro $\mu \mathrm{g} / \mathrm{ml}$ of cordycepin begins to approach that of the control. Although it is not indicated in this figure, the growth is about the same as that for the control after $30 \mathrm{~h}$.

Although the extent of inhibition was not as pronounced, this growth inhibitory effect was also noted when cordycepin was added under sterile conditions to cultures in the log phase of growth, Fig. 3.

The structural similarity of cordycepin to adenosine or deox yadenosine suggested the possibility that this compound might be functioning as an anti-metabolite and interfering with nucleic acid synthesis. To test this hypothesis, various compounds were added to cultures along with cordycepin in an attempt to prevent the inhibition. The results of such an experiment are given in Table $\mathrm{I}$. Of a number of compounds tested, adenine and guanine as their nucleoside derivatives were most effective. The addition of casein hydrolysate caused a stimulation of $B$. subtilis growth, but cordycepin still remained an equally effective inhibitor in this supplemented media. The separate addition of D-xylose, D-ribose, L-rhamnose, D-mannose, D-fructose, and cordycepose, at a final concentration of $10 \mu \mathrm{g} / \mathrm{ml}$, to cordycepin-inhibited cultures of $B$. subtilis did not result in a significant reversal of the growth-inhibiting effect. AMP and GMP, which have been shown to reverse growth inhibition caused by 


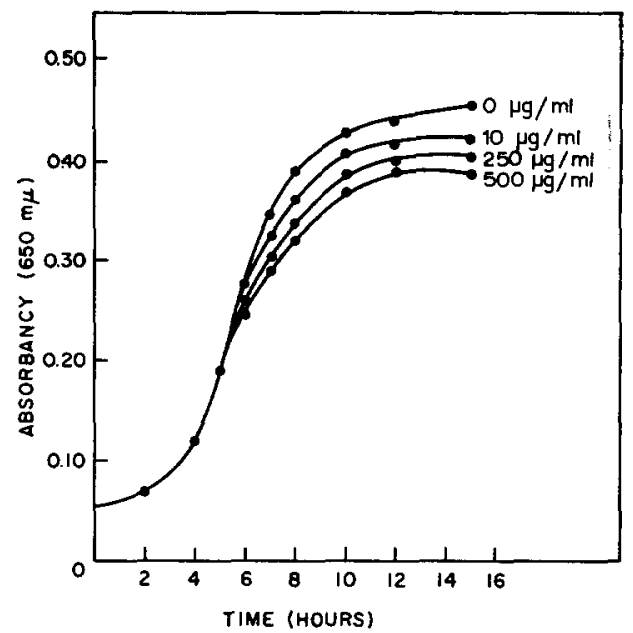

Fig. 3. Interruption of B. subtilis growth by cordycepin. Growth was followed turbidimetrically as described in the text. Cordycepin was added under sterile conditions $5 \mathrm{~h}$ post innoculation at the final concentrations indicated in the figure.

\section{TABLE I}

RELIEF OF CORDYCEPIN INHIBITION BY PURINES, PYRIMIDINES, AND THEIR NUCLEOSIDE DERIVATIVES

The relative efficiency of various compounds in relieving cordycepin inhibition was determined as described in the text. In each case, cordycepin was present in inhibited cultures at a final concentration of $100 \mu \mathrm{g} / \mathrm{ml}$ while the compound being tested was added at a final concentration of Io $\mu \mathrm{g} / \mathrm{ml}$.

\begin{tabular}{lc}
\hline \multicolumn{1}{c}{ Additions } & $\begin{array}{c}\text { Relief of } \\
\text { inhibition } \\
(\%)\end{array}$ \\
\hline Guanosine & 74 \\
Adenosine & 72 \\
Deoxyadenosine & 64 \\
Deoxyguanosine & 59 \\
Adenine & 55 \\
Inosine & 35 \\
Guanine & 33 \\
Deoxyinosine & $\mathrm{I} 6$ \\
Hypoxanthine & $\mathrm{I} 4$ \\
Xanthine & $\mathrm{I} 4$ \\
Cytidine & $\mathrm{I} 2$ \\
Xanthosine & 8 \\
Uridine & 8 \\
\hline
\end{tabular}

psicofuranine in $S$. aureus ${ }^{7}$, were found to be without effect in reversing cordycepin inhibition of $B$. subtilis growth. This might be explained by the inability of these nucleotides to gain entrance into the cell. The relief of inhibition shown by the various compounds listed in Table I was obtained from data similar to that illustrated for adenosine in Fig. 4. The amount of cordycepin used in each case was $100 \mu \mathrm{g} / \mathrm{ml}$ while the compound being tested was added at a concentration of $r o \mu \mathrm{g} / \mathrm{ml}$. The slope of the log phase portion of the growth curve was compared to the corresponding 


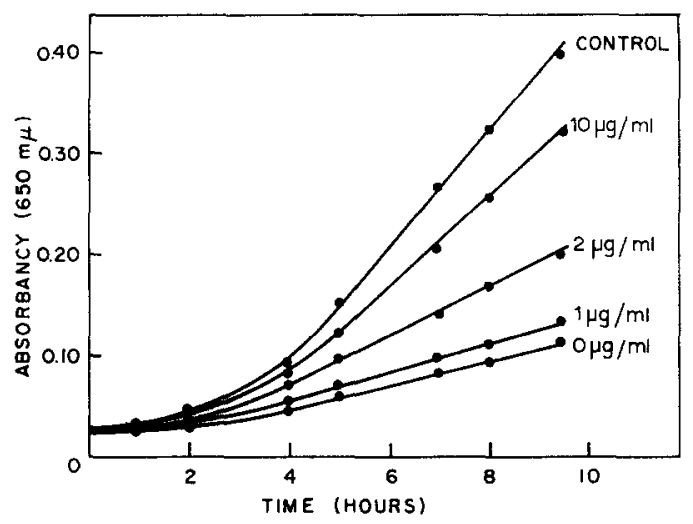

Fig. 4. Relief of cordycepin inhibition by adenosine. This experiment was carried out as described in the text. The concentration of cordycepin in each case was $100 \mu \mathrm{g} / \mathrm{ml}$ except for the control which contained none. The final concentration of adenosine present in each vessel is indicated in the figure. Both adenosine and cordycepin were added prior to sterilization.

slope in the curve for the uninhibited culture. The ratio of these two slopes gave the relative efficiency of a compound in relieving inhibition and is expressed as the percent relief of inhibition.

The reversal of cordycepin inhibition by adenosine was studied further and the relationship was found to be competitive. The amount of growth observed in the presence of both cordycepin and adenosine is proportional to the ratio of their concentrations rather than the absolute amount of either nucleoside. This is illustrated in Fig. 5 .

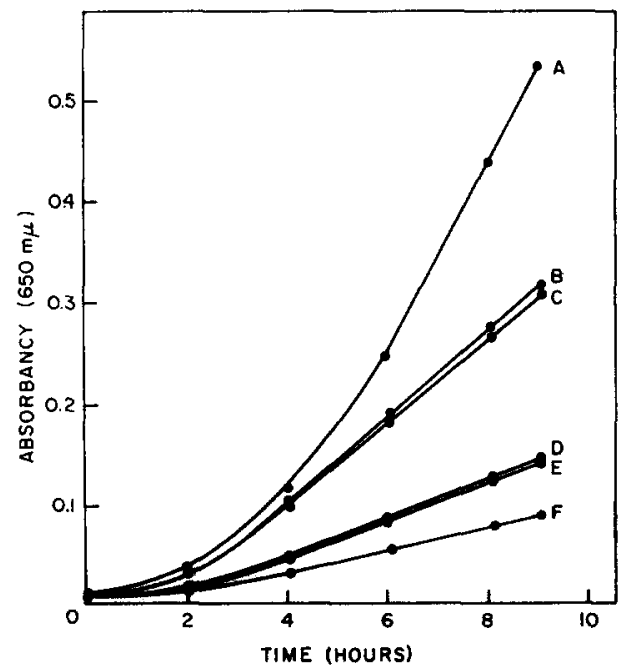

Fig. 5. Competitive relief of cordycepin inhibition by adenosine. The growth of cells was followed as described in the text. Vessel $A$, no additions. $B, 50 \mu \mathrm{g} / \mathrm{ml}$ cordycepin and $2.5 \mu \mathrm{g} / \mathrm{ml}$ adenosine. $\mathrm{C}, 100 \mu \mathrm{g} / \mathrm{ml}$ cord ycepin and $5.0 \mu \mathrm{g} / \mathrm{ml}$ adenosine. $\mathrm{D}, 100 \mu \mathrm{g} / \mathrm{ml}$ cordycepin and $\mathrm{I} .0 \mu \mathrm{g} / \mathrm{ml}$ adenosine. $\mathrm{E}, 200 \mu \mathrm{g} / \mathrm{ml}$ cordycepin and $2.0 \mu \mathrm{g} / \mathrm{ml}$ adenosine. F, roo $\mu \mathrm{g} / \mathrm{ml}$ cordycepin. Both compounds were added prior to sterilization. 
Two hypotheses can be presented to explain the fact that purine nucleosides are able to reverse the growth inhibitory effect of cordycepin. The first is that this compound could be interfering with reactions by which purines are interconverted once they are formed, or secondly, it may be interfering with reactions by which purines are synthesized de novo. To test the latter possibility, 5-amino-4-imidazolecarboxamide was tested for its ability to reverse the cordycepin effect. The ratio of cordycepin concentration to 5-amino-4-imidazolecarboxamide concentration required to achieve a $50 \%$ relief of inhibition was found to be approximately I : Io whereas the ratio of cordycepin to adenosine required to achieve a $70 \%$ relief of inhibition was ro:I. The fact that 5-amino-4-imidazolecarboxamide supports the growth of purine-requiring bacterial mutants, but at much higher concentrations than required for intact purines ${ }^{8}$ is consistant with the high concentrations necessary to prevent cordycepin inhibition.

The ability of 5-amino-4-imidazolecarboxamide to overcome inhibition seems to support the concept that cordycepin is blocking some step prior to the formation of the complete purine ring. If the interconversion of nucleotides was the primary site of cordycepin action, then the addition of a precursor which is common to all nucleotides should not relieve the inhibition.

Replacement of the 6-amino group of cordycepin by a hydroxyl group produced the hypoxanthine analog of cordycepin. When this compound was tested for growth inhibition, it was found to be effective. However, it required much higher concentrations to produce the same degree of inhibition as obtained with the parent compound (Fig. 6).

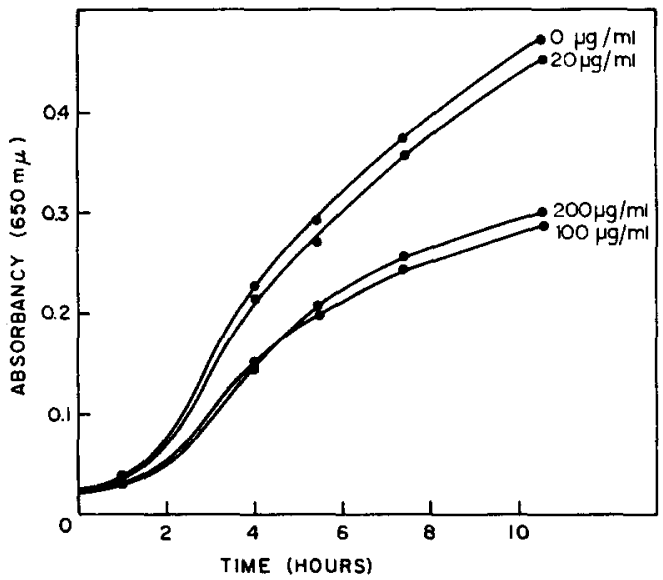

Fig. 6. Inhibition of $B$. subtilis growth by the hypoxanthine analog of cordycepin. The inhibitor was added before sterilization, otherwise incubation and growth were followed as described in the text. The final concentration of inhibitor present is indicated in the figure.

As was noted earlier, $B$. subtilis is capable of gradually overcoming the growth inhibitory effect of cordycepin at low concentrations of the inhibitor. Since adenine reverses the growth inhibitory effect (Table I), a possible explanation of this phenomenon might be the ability of the organism to cleave cordycepin giving adenine and cordycepose, and the gradual accumulation of adenine at the expense of the inhibitor allows for some growth. To test this hypothesis, freshly grown cells were 
harvested in the log phase of growth, washed with sterile Herriott's media, and divided into two equal portions. In one case, cells were resuspended in fresh media containing no additions; in the other, the same media containing $\left[8-{ }^{14} \mathrm{C}\right]$ cordycepin (specific activity 200 counts $/ \mathrm{min} / \mu$ mole) at a concentration of $100 \mu \mathrm{g} / \mathrm{ml}$ was used to resuspend the cells. After $3 \mathrm{~h}$ incubation, the cells were removed by centrifugation. The clear supernatant fluids were treated with charcoal until approx. $90 \%$ of the ultraviolet-absorbing material was removed from the solution. The charcoal was filtered off and treated twice with $20 \%$ aqueous pyridine. The pyridine eluates were combined and concentrated to dryness in a rotary-film evaporator under vacuum. After the addition of water, this material was concentrated to dryness a number of times to insure the complete removal of pyridine.

The residues were dissolved in water and made $0.04 \mathrm{M}$ with sodium borate. The $\mathrm{pH}$ of the solution was adjusted to I I with $\mathrm{NaOH}$ and the material was placed on a Dowex-I $\left(\mathrm{Cl}^{-}\right)$column $\left(2 \times \mathrm{I}_{5} \mathrm{~cm}\right)$. The resin column was treated with $0.0 \mathrm{I} \mathrm{M}$ sodium borate ( $\mathrm{pH}$ Io), until the absorbancy of the effluent was below o.I. Further treatment of the resin column with $0.2 \mathrm{~N} \mathrm{NaCl}$ in o.or $\mathrm{N} \mathrm{HCl}$ eluted a series of ultravioletabsorbing compounds which were adsorbed on charcoal and subsequently eluted with $20 \%$ aqueous pyridine. After removal of the pyridine as before, the eluates were chromatographed on paper by descending chromatography using water adjusted to $\mathrm{pH}$ Io with aqueous ammonia as the resolving fluid. The substances present on the chromatograms were visualized with an ultraviolet lamp and the compounds observed were eluted and their identity confirmed by their absorption spectra in acid and alkali.

In both experiments, the media in which the cells were grown contained a number of ultraviolet-absorbing substances. However the media to which cordycepin had been added contained free adenine (specific activity of Igo counts $/ \mathrm{min} / \mu$ mole) and hypoxanthine (specific activity of 250 counts $/ \mathrm{min} / \mu \mathrm{mole}$ ) which were not present in the control media. This indicated that the two bases were derived rather directly from cordycepin since their specific activities were close to that of the added cordycepin. Whether the process of deamination forming hypoxanthine occurred at the nucleoside or free base level is not known at this time.

Attempts were made to detect the accumulation of an intermediate both in the media and trichloroacetic acid-extractable fraction of cordycepin-inhibited cells. The material so obtained was fractionated on a resin column of Dowex- $\left(\mathrm{Cl}^{-}\right)$by a concave gradient elution technique, and in no case did we observe the accumulation at the nucleoside or nucleotide level of an ultraviolet-absorbing compound not present in comparable amounts in control cells or media. If cordycepin were blocking the conversion of a specific nucleotide, one might expect that nucleoside derivative in the media, if the equilibrium of the preceding reaction was favorable. Such an observation was made in psicofuranine inhibition where xanthosine was found to accumulate in the media of cells grown in the presence of the inhibitor ${ }^{9}$.

An inhibition of nucleotide phosphorylation to the di- and triphosphate derivatives should be reflected in the acid-soluble fraction also. Since these changes were not observed, these results are compatible with the suggestion that the metabolic block occurs at some stage prior to the completion of the intact purine ring system. The fact that 5-amino-4-imidazolecarboxamide has the ability to overcome this inhibition, although rather poorly, also is in agreement with this view. Further 
work along these lines involves studying the incorporation of radioactivie precursors into the nucleic acid purines of this organism in the presence and in the absence of cordycepin. So far, such experiments support the concept that the metabolic block occurs prior to completion of the purine ring.

\section{ACKNOWLEDGEMENTS}

This work is taken in part from a thesis submitted by F. RotTMAN to the Horace $H$. Rackham School of Graduate Studies in partial fulfillment for the degree, Doctor of Philosophy.

This work was supported in part by a research grant from the United States Public Health Service (CA-0522I) and by a predoctoral Public Health Service Fellowship to F. Rottman (No. GF I7, I08) from the Division of General Medical Sciences, Public Health Service.

\section{REFERENCES}

1 K. G. Cunningham, S. A. Hutchinson, W. Manson and F. S. Spring, J. Chem. Soc., (r95i) 2299.

2 D. V. Jagger, N. M. Kredich and A. J. Guarino, Cancer Res., 2 I (I96I) 2 I6.

H. Klenow, Biochem. Biophys. Res. Commun., 5 (I96r) I 56.

- N. M. Kredich and A. J. Guarino, Biochim. Biophys. Acta, 4I (rg6o) 363.

- P. A. Levene and R. S. Tipson, J. Biol. Chem., i i I (1935) 3 I 3 .

- R. M. Herrioti and J. L. Barlow, J. Gen. Physiol., 36 (1952) I7.

? L. J. Hanka, J. Bacteriol., 80 (I960) 30.

8 J. S. Gots, Arch. Biochem. Biophys., 29 (1950) 222.

- L. Slechta, Biochem. Pharmacol., 5 (1960) 96. 